\title{
Primary Voltage Control in Active Distribution Networks via Broadcast Signals: The Case of Distributed Storage
}

\author{
Konstantina Christakou, Student Member, IEEE, Dan-Cristian Tomozei, Member, IEEE, Maryam Bahramipanah, \\ Jean-Yves Le Boudec, Fellow, IEEE, and Mario Paolone, Senior Member, IEEE
}

\begin{abstract}
In this paper, we consider an active distribution network (ADN) that performs primary voltage control using real-time demand response via a broadcast low-rate communication signal. The ADN also owns distributed electrical energy storage. We show that it is possible to use the same broadcast signal deployed for controlling loads to manage the distributed storage. To this end, we propose an appropriate control law to be embedded into the distributed electrical storage controllers that reacts to the defined broadcast signal in order to control both active and reactive power injections. We analyze, in particular, the case where electrical storage systems consist of supercapacitor arrays and where the ADN uses the grid explicit congestion notification (GECN) for real-time demand response that the authors have developed in a previous contribution. We estimate the energy reserve required for successfully performing voltage control depending on the characteristics of the network. The performance of the scheme is numerically evaluated on the IEEE 34-node test feeder. We further evaluate the effect, depending on the line characteristics, of reactive versus active power controlled injections. We find that without altering the demand-response signal, a suitably designed controller implemented in the storage devices enables them to successfully contribute to primary voltage control.
\end{abstract}

Index Terms-Active distribution network (ADN), ancillary services, broadcast signals, demand response, electrical energy storage systems, primary voltage control.

\section{INTRODUCTION}

$\mathbf{T}$ HE increasing penetration of distributed generation in distribution networks, essentially composed by nondispatchable resources, renders the control of these networks compelling and calls for active control mechanisms in order to achieve specific operation objectives (e.g., [1]-[7]). In particular, the grid ancillary services ${ }^{1}$ typically employed in the HV transmission networks are expected to be extended to distribution networks,

Manuscript received August 26, 2013; revised December 16, 2013 and February 18, 2014; accepted April 12, 2014. Date of publication August 19, 2014; date of current version September 05, 2014. Paper no TSG-00697-2013.

The authors are with the Ecole Polytechnique Fédérale de Lausanne, CH-1015 Lausanne, Switzerland (e-mail: konstantina.christakou@epfl.ch; dan-cristian.tomozei@epfl.ch; maryam.bahramipanah@epfl.ch; jean-yves. leboudec@epfl.ch; mario.paolone@epfl.ch).

Digital Object Identifier 10.1109/TSG.2014.2319303

${ }^{1}$ By "grid ancillary services" we refer to frequency support, voltage support, black start and island operation capabilities, system coordination, and operational measurement. See, as a general reference, [8] for further details. as was recently proposed by the European Network of Transmission System Operators for Electricity (ENTSO-E) [8].

With the increasing availability of communication technologies, we envision that, in distribution networks, these types of ancillary services can be provided by distributed and controllable energy resources such as generators, loads and energy storage systems. For instance, in [5] the optimal scheduling of generators is proposed for voltage control and minimization of the losses in the network. ${ }^{2}$ Furthermore, forecast uncertainties and increased volatility in the renewable energy production can be tackled by means of local distributed energy storage systems or elastic loads (e.g., [9] and [10]).

Most such control schemes rely on two-way communication between the controllable entity and the distribution network operator (DNO) (e.g., [11] and [12]). However, the distributed nature of the controllable resources, as well as their large number and small individual impact, motivates the use of control mechanisms based on one-way communication. In [13], for instance, the charging rate of electric vehicles is controlled via broadcast signals so as to avoid overloading the distribution feeders. Furthermore, the authors in [14] propose the use of a universal broadcast signal to control the charge rate of a fleet of electric vehicles for the local compensation of renewable production volatility.

The purpose of this work is twofold. On one hand, we evaluate the potential of distributed energy storage systems (ESSs) for providing primary voltage control via broadcast signals, computed as in [15] for the control of thermal loads. On the other hand, we investigate the possibility of using the same broadcast signal in order to control heterogeneous energy resources (e.g., ESSs and loads). ESSs are selected as the targeted energy resources because they are expected to cover a wide spectrum of applications in distribution networks. They are characterized by charge/discharge cycles that could range from seconds (typically in high-power applications) to hours or even days (in high-energy applications) [16]. As a consequence, ESSs are able to compensate instantaneous imbalances (e.g., fluctuations of renewable generation), to time-shift the energy production or consumption (e.g., slow variations in renewable generation), and to contribute to voltage support (e.g., [17] and [18]).

\footnotetext{
${ }^{2}$ Although the minimization of the losses does not explicitly solve the problem of line congestion, it provides a solution that tends to be in the same direction as congestion alleviation.
} 
In this paper, we assume that ESSs are used to provide ancillary services to the medium voltage grid. They are assumed to be indirectly controlled by the DNO via real-time demand-response (DR) broadcast signals. We consider that these signals are computed by using the "grid explicit congestion notification mechanism" (GECN) control mechanism ([15]), which we recall briefly in Section II. In the original setting of [15], the control signal is meant for elastic loads that consume mostly active power. As the ESSs can provide both active and reactive power support to the grid, we send an additional broadcast signal meant for explicitly controlling reactive power injections. Thus, the controller of the loads remains the same (it simply disregards the reactive power signal). We propose a controller design for the ESSs that reacts to both signals, and is tailored to the characteristics of the considered storage devices.

Within the context of voltage control in active distribution networks (ADNs), it is important to point out that both active and reactive power-injection controls play an important role for this specific ancillary service, in view of the nonnegligible $R / X$ ratio of longitudinal parameters of the medium and low voltage lines (see Section IV-D, but also [19] and [20]). With this in mind, we first evaluate the ESSs sizing required to improve the network voltage profiles. Finally, we investigate the performance of the considered real-time mechanism when mixed populations of controllable resources with different characteristics (e.g., size, inertia, and storage capabilities) are present in the network.

The paper is structured as follows. Section II gives the necessary background on primary voltage control via broadcast signals. Section III focuses on the representation of storage devices and on the description of a specific type of ESS, namely supercapacitors. It also describes the proposed ESS controller and includes a discussion concerning the approximate sizing of these devices. In Section IV, the evaluation of the proposed scheme is provided through application examples using the IEEE 34-node test feeder where supercapacitor arrays, as well as thermostatically controlled appliances are present in each network bus. Section $\mathrm{V}$ provides a discussion on the comparison of the proposed mechanism to traditional control methods and its application for the control of heterogeneous populations of energy resources. Finally, Section VI concludes the paper with the final remarks on the benefits and the applicability of the method and with possible future applications.

\section{Primary Voltage Control Via Broadcast Signals}

In this section, we describe briefly the principles and operations of the GECN control mechanism proposed in [15]. This mechanism acts on a fast time-scale and is designed to provide ancillary services to the grid by means of low bit-rate broadcast control signals. In order to provide primary voltage control, GECN relies on the assumption that the DNO manages the consumption of a large population of small dispersed resources in the network. In this work we evaluate the possibility of using the same GECN signal for controlling a heterogeneous population of resources, namely:

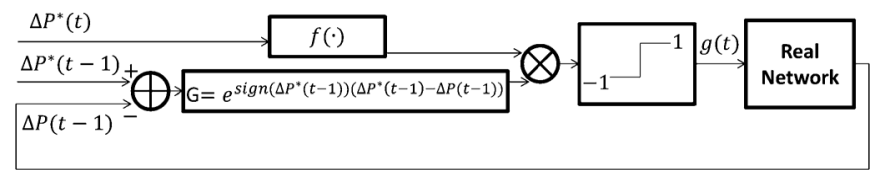

Fig. 1. Control loop for the computation of the GECN signal $\mathbf{g}(t)$ for the control of active power. Adapted from [15].

- flexible loads, for which we control only the consumption (mostly active power), subject to state constraints (such as a temperature deadband);

- energy storage units, which can absorb and inject both active and reactive power.

For the sake of presentation clarity, we do not consider the control of centralized resources, such as transformers tap changers. However, we note that their inclusion in the control loop is straightforward [15].

Based on real-time measurements, a state estimator provides, at each time step, to the DNO the state of the network in each bus $i$ : injected/absorbed active power $P_{i}(t)$, reactive power $Q_{i}(t)$, as well as voltage phasors $\bar{E}_{i}(t)$. The rated value of the voltage in the network is denoted by $E_{n}$. At the next time step, the DNO needs to match as closely as possible the scheduled consumption profile $\left(P_{i}^{f}(t+1), Q_{i}^{f}(t+1)\right)$, while maintaining the system within acceptable operating bounds in terms of voltage magnitude $E_{n}-\delta \leq\left|\bar{E}_{i}(t+1)\right| \leq E_{n}+\delta$. We consider that, in absence of control, the mismatch in bus $i$ is $\Delta P_{i}^{f}(t):=P_{i}^{f}(t+1)-P(t)$ and $\Delta Q_{i}^{f}(t):=Q_{i}^{f}(t+1)-Q(t)$. The DNO computes desired power adjustments $\left(\Delta P_{i}(t), \Delta Q_{i}(t)\right)$ in buses $i$ equipped with controllable resources and broadcasts appropriate control signals. However, controllable resources react depending on their internal state.

The implementation of the scheme is based on the closedloop control depicted in Fig. 1. At each time step, the DNO computes the voltage sensitivity coefficients with respect to absorbed/injected power of a network bus $\ell$ :

$$
K_{P, i \ell}(t):=\frac{\partial\left|\bar{E}_{i}(t)\right|}{\partial P_{\ell}}, K_{Q, i \ell}(t):=\frac{\partial\left|\bar{E}_{i}(t)\right|}{\partial Q_{\ell}}
$$

for instance, by solving the linear systems of equations presented in [20], [21]. This allows for a local linearization of the voltage deviation $\Delta|\overline{\mathbf{E}}(t)|=\left(\Delta\left|\bar{E}_{i}(t)\right|\right)_{i}$ :

$$
\Delta|\overline{\mathbf{E}}(t)| \approx \mathbf{K}_{\mathbf{P}}(t) \Delta \mathbf{P}(t)+\mathbf{K}_{\mathbf{Q}}(t) \Delta \mathbf{Q}(t) .
$$

Next, the DNO computes the optimal required power adjustments $\left(\Delta \mathbf{P}^{*}(t), \Delta \mathbf{Q}^{*}(t)\right)$ in the buses, which lead to the desired operation set point for voltage control by solving the constrained optimization problem:

$$
\begin{aligned}
& \min _{\Delta \mathbf{P}, \Delta \mathbf{Q}} \sum_{i} \mu_{i}\left(\Delta P_{i}(t)-\Delta P_{i}^{f}(t)\right)^{2} \\
&+\sum_{i} \mu_{i}\left(\Delta Q_{i}(t)-\Delta Q_{i}^{f}(t)\right)^{2} \\
&+\sum_{i} \lambda_{i}\left[\left(\left|\bar{E}_{i}(t)\right|+\Delta\left|\bar{E}_{i}(t)\right|-E_{n}\right)^{2}-\delta^{2}\right]^{+} \\
& \text {subject to }: \quad \gamma_{i} \leq \cos \varphi_{i} \leq 1
\end{aligned}
$$


where $\gamma_{i}$ is the constraint on the power factor, $\cos \varphi_{i}$, of the $i$ th bus and the first two terms in the objective function are weighted by parameters $\lambda_{i}$ and $\mu_{i}{ }^{3}$

Finally, the resulting optimal set points, $\left(\Delta \mathbf{P}^{*}(t), \Delta \mathbf{Q}^{*}(t)\right)$ are mapped to a signal $g(t)=\left(g_{P, i}(t), g_{Q, i}(t)\right)_{i}$ with components in the range $[-1,1]$ corresponding to active and reactive power adjustments in each bus $i$. For both active and reactive power, a negative $g$ encourages consumption, a positive $g$ inhibits consumption, and $g=0$ does not affect the behavior of the controllable resources. Finally, the resulting variation of the aggregate injected/absorbed power at the buses provides the DNO with an implicit feedback with respect to the responsiveness of the bus resources. This variation plays a role in deciding the control actions for subsequent time steps.

In Fig. 1, we detail this feedback loop for the active power broadcast signal $g_{P}$. A similar closed-loop controller is adopted for the reactive power. In this way broadcast signals can be computed for both power set points. At time $t, g_{P}(t)$ is computed as a function of 1) the optimal set points at the current time-step and 2) the mismatch $G$ between the optimal and the actual set points that the DNO observed at the previous time step $t-1$.

The various distributed resources in network bus $i$ receive the broadcast signal $\left(g_{P, i}, g_{Q, i}\right)$. The local controller of a certain resource attached to this bus decides the action to be taken based on the internal state of the resource and on the value of the received signal. For example, in [15] a refrigerator controller reacts to a $g_{P}$ signal only if it has not already done so in the near past, at most a predetermined number of time steps ago. This ensures that operation in mini-cycles is avoided. Next, if this first test is passed, the controller takes the decision of turning on or off the refrigerator with a certain probability that depends on the signal and on the internal state of the refrigerator (i.e., its internal temperature). All the details concerning this specific aspect are given in [15].

In this work, we are interested in controlling distributed electrical storage, in addition to flexible loads; in order to keep the system tractable, we would like to avoid individual point-to-point communication, from the DNO's controller to every storage system. It is thus natural to use broadcast signals and to rely on state estimation for the feedback channel, as with GECN. We go one step further and ask whether the same GECN signal can be used for controlling together the ESSs and the flexible loads (refrigerators). We show that this is indeed possible, without any change to GECN, by implementing an appropriate control law in the ESS controllers. In other words, we propose that the same GECN signals are broadcast to the different buses of the network; 4 it is the local controller of each elastic appliance or storage system that decides the system's response to the received signal. In the case of DR, it is assumed that the elastic loads consume an amount of active power and the corresponding proportional reactive power, obtained

\footnotetext{
${ }^{3}$ The choice of the weights in the objective function is related to the topology of the network and the parameters of the lines (i.e., the network admittance matrix).

${ }^{4}$ Note that even though the same GECN signals are used to control loads and ESSs, different GECN signals are sent to the different network buses. This allows controllability even in cases when different network buses have completely different voltage profiles during the day.
}

via the power factor. Therefore, elastic loads react to only one broadcast signal $\left(g_{P}\right)$ that is used to control their power consumption. On the contrary, in the case of ESS control, both broadcast signals $\left(g_{P}\right.$ and $\left.g_{Q}\right)$ are used to control both active and reactive power.

In the following section, we briefly present the model of a given storage device used in the rest of the paper, as well as the design of a controller suitable for its contribution to primary voltage control. ${ }^{5}$

\section{ELECTROCHEMiCAL ENERGy StORAGE REPRESENTATION AND CONTROL}

In this section, the general representation of electrochemical energy storage systems is presented and a controller, tailored to the characteristics of supercapacitors, is proposed.

\section{A. General Formulation of the State-of-Charge of Electrochemical-Based Storage Systems}

The estimation of the so-called state of charge $(S o C)$ of an electrochemical-based storage system is of great importance in the majority of applications dealing with operation and control of electrochemical ESSs [22].

Several methods, that use different criteria in order to estimate the $S o C$, are proposed in the literature. As discussed in [23], the five most important criteria, with particular reference to batteries, are 1) measurement of electrolyte specific gravity, 2) battery current time-integration, 3) battery impedance/resistance estimation, 4) measurement of the battery open circuit voltage, and 5) inclusion of electrolyte temperature, discharge, rate and other battery parameters. A general equation, that defines the $S o C$ at a specific time instant and is a combination of the above criteria, is (e.g., [22]-[26]):

$$
S o C(t)=\frac{C\left(t_{0}\right)-\alpha(I, \theta) \int i(t) d t}{C(I, \theta)}
$$

where $C(I, \theta)$ is the ESS capacity for a constant current discharge rate $I$ at electrolyte temperature $\theta, C\left(t_{0}\right)$ is the ESS capacity at time $t_{0}, i(t)$ is the instantaneous value of the current and $\alpha$ is the charge-efficiency coefficient associated to charge and discharge phases. ${ }^{6}$

In this work, the $S o C$, computed as in (4), will be incorporated by the storage controller as better discussed in Section III-C.

\section{B. Circuit-Based Model of Electrochemical ESS Applied to the Case of Supercapacitors}

A general approach in modeling electrochemical ESSs is to represent a single cell with an equivalent circuit-based model that simulates their behavior (e.g., [27]-[29]). These models provide simple structures that can represent sufficiently the dynamic behavior of these ESSs as they are directly related to the

\footnotetext{
${ }^{5}$ The model and the local controller design for thermostatically controlled appliances are omitted as they are described in detail in [15].

${ }^{6}$ As a first approximation $\alpha(I, \theta)$ can be assumed equal to 1 . Specific tests on the targeted storage systems can infer this function.
} 


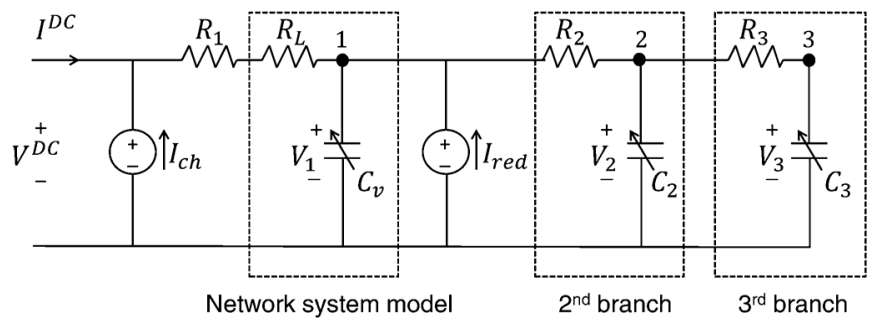

Fig. 2. Proposed SC model in [33].

physics/chemistry of the cell configuration. The major advantage of this approach is that the relationship between the cell voltage and the current drawn or supplied to the cell can often be expressed analytically by solving a system of ordinary differential equations [30].

In this paper, supercapacitors (SC) have been selected as the targeted ESS. Due to their high power density, short charge time, and long life duration, these devices are particularly interesting in the ESS applications that require cycles with high dynamics (e.g., primary voltage control via fast compensation of renewable DG, fast charging of electric vehicles) [31]. Several circuit-based models, which can represent the SC behavior in both steady-state and dynamic conditions, are proposed in the literature (e.g., [32] and [33]). In this work, the model developed in [33] is considered (see the circuit model shown in Fig. 2). This model enables to correctly represent both the quasi-static and dynamic behavior of a SC accounting for the so-called "redistribution-effect" that plays a major role in the SC dynamic response.

For this specific model, the $\mathrm{SC}$ terminal voltage, $V^{\mathrm{DC}}$, is linked to the input current, $I^{\mathrm{DC}}$, via the following system of ordinary differential equations:

$$
\begin{aligned}
\frac{d V_{1}}{d t} & =\left(-\left(I^{\mathrm{DC}}+I_{c h}\right)+\frac{V_{1}-V_{2}}{R_{2}}+V_{1} \frac{d C_{v}}{d t}-I_{r e d}\right) \frac{-1}{C_{v}} \\
\frac{d V_{2}}{d t} & =\left(\frac{V_{2}-V_{3}}{R_{3}}-\frac{V_{1}-V_{2}}{R_{2}}+V_{2} \frac{d C_{2}}{d t}\right) \frac{-1}{C_{2}} \\
\frac{d V_{3}}{d t} & =\left(-\frac{V_{2}-V_{3}}{R_{3}}+V_{3} \frac{d C_{3}}{d t}\right) \frac{-1}{C_{3}} \\
V^{\mathrm{DC}} & =V_{1}+\left(I^{\mathrm{DC}}+I_{c h}\right)\left(R_{1}+R_{L}\right)
\end{aligned}
$$

where $R_{1}$ is the input electrode resistance; $R_{L}$ and $C_{v}$ are the resistance and the capacitance of the so-called "SC network system model" and; $R_{2}, C_{2}$ and $R_{3}, C_{3}$ are the resistances and the capacitances of the second and third branch, respectively. All the capacitances exhibit a nonlinear dependence on the voltage. This dependence is taken into account by curve-fitting measurements obtained via experimental tests. As proposed in [33], the two current sources, $I_{c h}$ and $I_{r e d}$, allow for improving the dynamics of the $\mathrm{SC}$ by taking into account the diffusion of the residual charge during the charge/discharge phases (short-time phenomenon), as well as during the redistribution phase (long-time phenomenon).

In the rest of the paper, we assume that $\mathrm{SC}$ cells are arranged in parallel and series connections suitable to form an array of a given total energy and power capacities. A bidirectional DC/AC converter is used to interface the $\mathrm{SC}$ with the network. The state of each cell is assumed to be its terminal voltage and the evolution of this state is described by (5). In order to model the power converter, the constraints on the $\mathrm{AC}$ active and reactive power should be taken into account. The $P Q$ capability curve of the converter is described by the following inequality constraint:

$$
\sqrt{\left(P^{\mathrm{AC}}\right)^{2}+\left(Q^{\mathrm{AC}}\right)^{2}} \leq S_{r}
$$

where $S_{r}$ is the rated power of the converter and $P^{\mathrm{AC}}, Q^{\mathrm{AC}}$ the active/reactive power flows on the $\mathrm{AC}$ side of the power converter interfacing the SC towards the grid.

It is assumed, as a first approximation, that the $\mathrm{DC} / \mathrm{AC}$ converter is characterized by an efficiency $(\eta)$ independent of its power flow. It is also assumed that this power converter can operate in four quadrants.

\section{Storage Controller}

In comparison with [15], where active power signals were used, the storage devices connected to a network bus $i$ receive at each time step $t$ two broadcast control signals, $g_{P}(t)$ for the active power and $g_{Q_{i}}(t)$ for the reactive power. Each signal represents a real number $g_{P_{i}}(t), g_{Q_{i}}(t) \in[-1,1]$. The control signals $g_{P_{i}}(t), g_{Q_{i}}(t)$ reflect the DNO's desire to inhibit (or encourage) consumption. Hence, a negative $g_{P_{i}}$ encourages charging, a positive $g_{P i}$ encourages discharging, and $g_{P i}=0$ does not have an effect on the storage devices. Similarly, a negative $g_{Q_{i}}$ calls for reactive power absorption, a positive $g_{Q_{i}}$ requests for more reactive power support, and $g_{Q_{i}}=0$ means that the DNO is satisfied with the current state of the ESS.

In the following, we propose a controller that takes into account these signals. As described, in response to nonzero $g_{P i}(t), g_{Q_{i}}(t)$ signals, the SC decides to charge or discharge an amount of energy. This decision is a function of the signals, the $S o C$, the DC voltage, as well as the previous state of the device. When this decision is made, the controller chooses the next state of the device as follows:

1) Upon receiving $g_{P i}(t)$ and $g_{Q_{i}}(t)$, the controller considers the signals as requested adjustments in its $\mathrm{AC}$-side active and reactive power set points expressed as fractions of the rated power:

$$
\begin{aligned}
& \Delta P_{i}^{\mathrm{AC}}(t)=-S_{i, r} g_{P i}(t) \\
& \Delta Q_{i}^{\mathrm{AC}}(t)=-S_{i, r} g_{Q_{i}}(t) .
\end{aligned}
$$

In other words, the two signals are viewed as proportional to the desired response of the resources requested by the DNO.

2) Once the required adjustments to the power set points are computed, the controller verifies that the constraints on the $P Q$ capability curve of the converter are respected. If this is not the case, $\Delta P_{i}^{\mathrm{AC}}(t)$ and $\Delta Q_{i}^{\mathrm{AC}}(t)$ are adjusted in such a way that the total power set point is the closest to the feasible region represented by (6). Fig. 3 shows an example where the requested set points lead the system to a state where the constraints of the converter are violated (point 2 in Fig. 3) and where an adjustment is required to a new state (point 3 in Fig. 3). If the size of the SC arrays or the capabilities of the converter are limited, then the requested power set points are expected to be quite frequently in the 
limits of the capability curve. The proposed adjustment is chosen in order to avoid staying on the same point in the boundary of the PQ capability curve once this limit is reached. ${ }^{7}$

3) The actual response of the device depends on the current operating point $\left(P_{i}^{\mathrm{AC}}(t), Q_{i}^{\mathrm{AC}}(t)\right)$, on the $\mathrm{SC}$ internal state $\left(V_{i}^{\mathrm{DC}}(t)\right)$ and on its state of charge $\left(S o C_{i}(t)\right)$. The new AC set points are computed as a moving average of the previous operating point and the requested operating set point filtered by a function of the $S o C$ :

$$
\begin{aligned}
P_{i}^{\mathrm{AC}}(t+1) & =\omega \beta_{P}\left(P_{i}^{\mathrm{AC}}(t)+\Delta P_{i}^{\mathrm{AC}}(t)\right)+(1-\omega) P_{i}^{\mathrm{AC}}(t) \\
Q_{i}^{\mathrm{AC}}(t+1) & =\omega \beta_{Q}\left(Q_{i}^{\mathrm{AC}}(t)+\Delta Q_{i}^{\mathrm{AC}}(t)\right)+(1-\omega) Q_{i}^{\mathrm{AC}}(t)
\end{aligned}
$$

where $\omega$ is a fixed gain and $\beta_{P}$ and $\beta_{Q}$ are variable gains that depend on the current $S o C$ of the SC. Specifically, for the active power $\beta_{P}=\left(1-S o C_{i}\right)^{2}$, when the device is charging $\left(P_{i}^{\mathrm{AC}}(t)>0\right)$, and $\beta_{P}=\left(S o C_{i}\right)^{2}$ when the device is discharging $\left(P_{i}^{\mathrm{AC}}(t)<0\right)$. For the reactive power, $\beta_{Q}=\left(S o C_{i}\right)^{2}$ regardless of the sign of the requested reactive power flow. ${ }^{8}$ This coefficient is used to filter the total power provided by the storage devices in order to smooth their response by accounting for their internal state.

4) The internal-state constraints of the storage device are finally taken into account. In particular, if the DC voltage has reached a specific minimum $\left(V_{\min }^{\mathrm{DC}}\right)$ or maximum $\left(V_{\max }^{\mathrm{DC}}\right)$ value, then the controller refuses to participate in the action to avoid the intervention of the maximum/minimum voltage relays always used in these types of systems to preserve the power electronics [23]. If the limits are not yet reached, the $\mathrm{AC}$ set points are transformed into $\mathrm{DC}$ power requirements and, subsequently, in charging/discharging current references as follows:

$$
\begin{aligned}
P_{i}^{\text {loss }}(t+1) & =\left(1-\eta_{i}\right) \sqrt{\left(P_{i}^{\mathrm{AC}}(t+1)\right)^{2}+\left(Q_{i}^{\mathrm{AC}}(t+1)\right)^{2}} \\
P_{i}^{\mathrm{DC}}(t+1) & =P_{i}^{\mathrm{AC}}(t+1)+P_{i}^{\text {loss }}(t+1) \\
I_{i}^{\mathrm{DC}}(t+1) & =\frac{P_{i}^{\mathrm{DC}}(t+1)}{V_{i}^{\mathrm{DC}}(t)}
\end{aligned}
$$

where $P_{i}^{\text {loss }}$ represents the losses in the $i$ th power converter. At this point the $V_{i}^{\mathrm{DC}}$ is continuously changing as a function of the charging/discharging current $I_{i}^{\mathrm{DC}}$ based on the model of the $i$ th ESS. For instance, in the case of supercapacitors, $V_{i}^{\mathrm{DC}}$ is updated based on (5). Then the current is updated so as to maintain the $P_{i}^{\mathrm{DC}}$ set point constant, until the controller receives the next GECN signals.

\section{On the Sizing of the ESSS}

The sizing of an ESS is intimately coupled with its control algorithm. In this respect, in this subsection we illustrate a possible procedure for sizing the distributed storage systems to

\footnotetext{
${ }^{7}$ It is worth observing that if the intercept on the line between points 1 and 2 on Fig. 3 is chosen as the adjusted point (instead of point 3), then once the limit of the converter is reached and the subsequent set points are also outside of the capability curve the controller will stay in the same set point for several time steps.

${ }^{8}$ Note that the request of the reactive power always drains energy from the SC through the losses in the converter regardless of the sign of the reactive power flow.
}

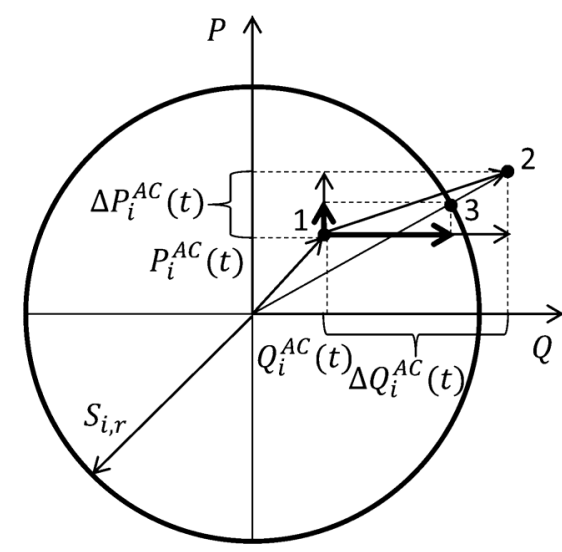

Fig. 3. Adjustment of the requested power set points in case of violation of the constraints of the $P Q$ capability curve of the converter.

fit the requests of the proposed control algorithm over a time window of $24 \mathrm{~h}$.

It is important to note that the sizing is done in terms of energy capacity. We describe below a method that, based on the observed forecast errors for a given day in the past, determines the required power adjustments from the storage system at each time step. The minimum energy capacity is deduced from these adjustments.

For the sizing procedure the DNO determines "the worst day" in terms of forecasting errors from historical data. For this day, the DNO has the imperfect 24-h per-bus forecasts for load and renewable profiles by $\left(\mathbf{P}^{f}(t), \mathbf{Q}^{f}(t)\right)$ and the actual per-bus measured power $(\mathbf{P}(t), \mathbf{Q}(t))$ and phase-to-ground voltage $\bar{E}_{i}(t)$. The DNO solves at each time step the optimization problem (3) and gets the process of optimal required power adjustments $\left(\Delta \mathbf{P}^{*}(t), \Delta \mathbf{Q}^{*}(t)\right)_{t}$ in the buses. These lead to the desired operation set points for voltage control for the whole 24-h period.

The solution of (3) provides profiles of $P Q$ set points for a given scenario of loads and distributed generation. Once the required power adjustments are computed for each bus, the DNO has a rough knowledge of the instantaneous amount of excess or deficit in the active and reactive power throughout the whole 24-h period. Thus, the DNO can compute the energy and, consequently, the size of storage devices that will be needed. The integral of the active power flow in each bus quantifies the required size for a given storage system. Nevertheless, the outcome of such a sizing remains related to the considered scenarios, and for this reason the presented method provides only an approximate sizing.

In our case, the targeted ESSs are SCs. Therefore, as they are characterized by high power density and low energy density, we take into account the nature of these devices and we do not utilize them for performing energy balance. To this end, we assumed as a worst case condition the one that involves large instantaneous errors in the forecasted PV power production. In particular, Fig. 4 shows the actual and forecasted daily aggregated profiles of active and reactive power of all the network buses used for the sizing of these devices, as well as the forecasting errors. 


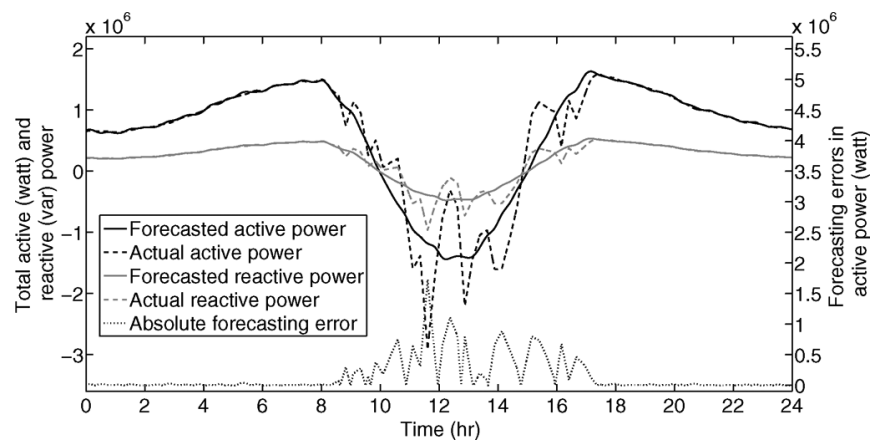

Fig. 4. Actual/forecasted values of aggregated active and reactive power injections of all network buses used for the sizing of the SC arrays.

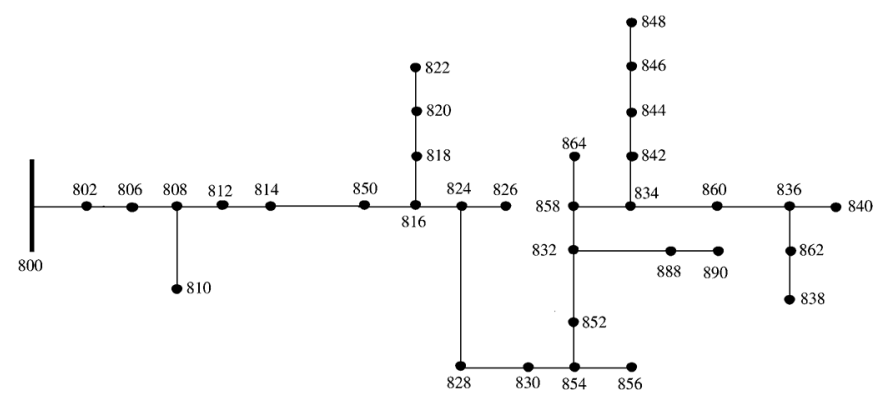

Fig. 5. Modified IEEE-34 node test feeder used for the evaluation of the proposed control mechanism [34].

\section{EVALUATION}

For the evaluation of the proposed mechanism we have considered a modified IEEE 34-node test feeder as depicted in Fig. 5. The modifications are 1) balanced lines and 2) the elimination of the regulators in line segments $814-850,852-832$, and of the shunt capacitors in buses 844,848 . The primary substation transformer is taken into account by considering its short-circuit internal impedance. The network load flow problem, the SC model (5), as well as the storage control mechanism are simulated in Matlab.

\section{A. Test Cases}

It is assumed that each network bus comprises a SC array, a large population of heterogeneous household controllable loads along with nonelastic demand, as well as non-dispatchable power injections. The elastic appliances consist of refrigerators modeled as in [15], whereas the nonelastic loads are represented by typical $24-\mathrm{h}$ curves. The main technical characteristics of the controllable appliances are given in Table I. Throughout the simulations, we have assumed that if an elastic appliance responds to a GECN signal by changing its mode of operation, then it will neglect the subsequent signals for a time window of 8 minutes to avoid operation in mini-cycles. Concerning the non-dispatchable power generation, we assume a PV-type profile with peak power that changes for all buses within the range of $60 \%-120 \%$ of each secondary substation peak load. As far as the forecasting errors are concerned, two different scenarios are considered. In the first scenario we assume a good 24-h-ahead forecast whereas in the second scenario we assume large forecasting errors. Fig. 6 shows the aggregate load profile of all 34 buses in the network for both test cases, where the convention used is that negative values represent power
TABLE I

PARAmeters of the Elastic APPliances AND the LOAD CONTROller

\begin{tabular}{|c|c|}
\hline Parameter & Value \\
\hline Temperature deadband, $\Theta\left({ }^{\circ} \mathrm{C}\right)$ & {$[3,8]$} \\
\hline Rated power, $P_{r}$ (Watt) & 150 \\
\hline Time step, $\tau(\mathrm{sec})$ & 1 \\
\hline Controller time counter, $T_{0}(\mathrm{sec})$ & 480 \\
\hline Appliance power factor, $\cos \varphi$ & 0.85 \\
\hline
\end{tabular}
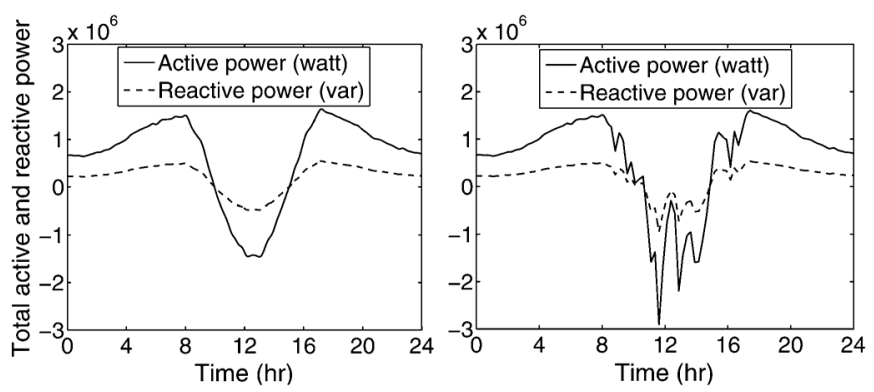

Fig. 6. Aggregate network active and reactive power profiles for two different scenarios of forecasting errors in the day-ahead PV production.

injection and positive power consumption. For the first scenario the peak values for the active and reactive power consumption shown in Fig. 6 are $1.64 \mathrm{MW}$ and 538 kvar, respectively. The corresponding peak value for the active power production of the distributed generation is $2.95 \mathrm{MW}$. For the second scenario the same load profiles are used, whereas the peak value for the active power production of the distributed generation is 4.24 MW.

\section{B. Storage System Sizing}

The SC arrays are sized approximately using the procedure described in Section III-D. To this end, in (3) the limit value of the power factor $\gamma_{i}$ in bus $i$ is set to 0.9 for all network buses, and the maximum voltage magnitude deviation $\delta$ is set to 0.04 (see [15] for further details). The number of cells in parallel connection, $N_{p}$, for each bus of the network is given in Table II. The number of cells in series, $N_{s}$, is equal to 149 for all buses. ${ }^{9}$ In the same table, we also provide the available energy of each array, as well as the rated power that limits the capabilities of the converter (6). The values of the energy reported in Table II are computed as the integral of the active power flows that resulted from the 24-h offline optimization described in Section III-D. It is worth observing that the amount of energy per bus required by ESSs to perform primary voltage control is in the order of few tens of $\mathrm{kWh}$. Such a limited reservoir appears compatible with a specific economic analysis of the use of SC. In the Appendix, we provide a brief economic analysis in order to illustrate the potential economic advantages of using SC versus Lithium-ion batteries for primary voltage control. The parameters of the storage controller, used hereafter, are given in Table III.

\section{Primary Voltage Control via Distributed Supercapacitors}

In this subsection, we evaluate the performance of the designed SC controller. To this end, the DNO employs the broadcast signals, $g_{P_{i}}$ and $g_{Q_{i}}$, described in Section III-C. The GECN

\footnotetext{
${ }^{9}$ The number of cells in series is determined by dividing the maximum DC voltage required, assumed here $400 \mathrm{~V}$, by the SC nominal cell voltage (i.e., $2.7 \mathrm{~V})$.
} 
TABLE II

Number of Parallel SC Cells, Rated Energy of ESS ANd Rated Power OF THE AC/DC CONVERTER PER NETWORK BUS

\begin{tabular}{|c|c|c|c|c|c|}
\hline Bus $\mathbf{N}_{\mathbf{o}}$ & $\mathbf{N}_{\mathbf{p}}$ & $\begin{array}{c}\text { Energy(kWh)/ } \\
\text { Power(MVA) }\end{array}$ & Bus $\mathbf{N}_{\circ}$ & $\mathbf{N}_{\mathbf{p}}$ & $\begin{array}{c}\text { Energy(kWh)/ } \\
\text { Power(MVA) }\end{array}$ \\
\hline 800 & - & - & 856 & 79 & $43.94 / 1.413$ \\
\hline 802 & 49 & $27.48 / 0.876$ & 852 & 82 & $45.61 / 1.466$ \\
\hline 806 & 53 & $29.16 / 0.948$ & 832 & 82 & $45.52 / 1.466$ \\
\hline 808 & 55 & $30.38 / 0.983$ & 888 & 84 & $46.98 / 1.502$ \\
\hline 810 & 55 & $30.54 / 0.983$ & 890 & 92 & $51.00 / 1.645$ \\
\hline 812 & 66 & $36.07 / 1.180$ & 858 & 82 & $45.60 / 1.466$ \\
\hline 814 & 68 & $37.03 / 1.216$ & 864 & 81 & $44.70 / 1.448$ \\
\hline 850 & 68 & $37.07 / 1.216$ & 834 & 92 & $50.31 / 1.645$ \\
\hline 816 & 68 & $37.99 / 1.216$ & 842 & 86 & $47.90 / 1.538$ \\
\hline 818 & 66 & $36.18 / 1.180$ & 844 & 90 & $49.45 / 1.609$ \\
\hline 820 & 66 & $36.82 / 1.180$ & 846 & 88 & $48.02 / 1.573$ \\
\hline 822 & 64 & $35.92 / 1.144$ & 848 & 88 & $48.18 / 1.573$ \\
\hline 824 & 75 & $41.99 / 1.341$ & 860 & 92 & $50.62 / 1.645$ \\
\hline 826 & 71 & $39.58 / 1.270$ & 836 & 90 & $49.54 / 1.609$ \\
\hline 828 & 75 & $41.17 / 1.341$ & 862 & 84 & $46.44 / 1.502$ \\
\hline 830 & 75 & $41.31 / 1.341$ & 838 & 86 & $47.41 / 1.538$ \\
\hline 854 & 75 & $41.51 / 1.341$ & 840 & 82 & $45.54 / 1.466$ \\
\hline
\end{tabular}

TABLE III

PARAMETERS OF THE STORAGE CONTROLLER

\begin{tabular}{|c|c|}
\hline Parameter & value \\
\hline Voltage deadband of single cell, $V$ DC $($ Volts) & {$[0.8,2.7]$} \\
\hline Capacity of single cell, $C_{c e l l}(\mathrm{~F})$ & 3600 \\
\hline Gain, $\omega$ & 0.2 \\
\hline Converter efficiency, $\eta$ & 0.95 \\
\hline
\end{tabular}

signals are computed and sent to the network buses each 16 seconds.

In order to infer the benefits of using distributed storage for primary voltage control, we show in Fig. 7 the initial voltage profile in the network, as well as the improvement due to the SC response for both test cases presented in Section IV-A. For the sake of brevity, we show the median value of the network voltages at every time step (solid line), along with the relevant $99 \%$ confidence intervals (dashed lines). In Scenario I the improvement in the voltage profile is in the order of $2 \%$. The largest advantage of the proposed control mechanism emerges in the case of large forecasting errors where the maximum improvement in the daily voltage profile is in the order of 6\%. In Fig. 8, the median value of the $S o C$ of the SC arrays is shown, as well as the relevant $99 \%$ confidence intervals.

Finally, we show in Fig. 9, the GECN signals for the active and reactive power sent to a single network bus. One can observe that when the forecasting errors are small, the request for reactive power is larger than the one for active power. As explained later, this is due to the ratio of $R / X$ of the network lines. Under large errors in the day-ahead PV production, however, the GECN signal adapts itself and becomes significantly larger for the active than for the reactive power.

\section{On the Adequacy of volt/var Control in ADNs}

Traditionally, voltage control is related to reactive power control (e.g., static var compensators) [35]. This is true in the case of HV transmission networks or, in general, networks where the ratio of the longitudinal-line resistance versus reactance is small and the decoupling of the active and reactive power is a valid

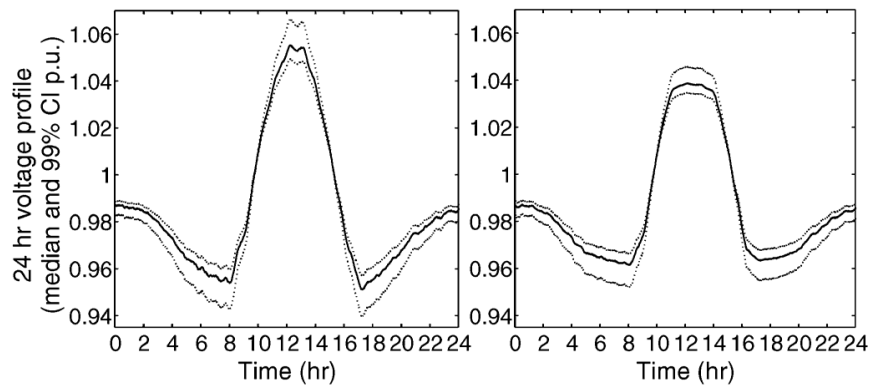

(a)
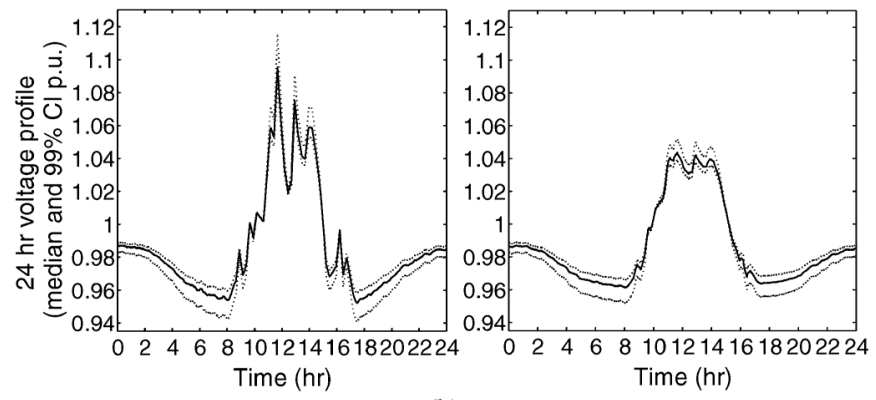

(b)

Fig. 7. Base case and improved 24-h network voltage profiles for two different scenarios of forecasting errors in the PV production. (a) Base case and improved voltage profiles for Scenario I. (b) Base case and improved voltage profiles for Scenario II.
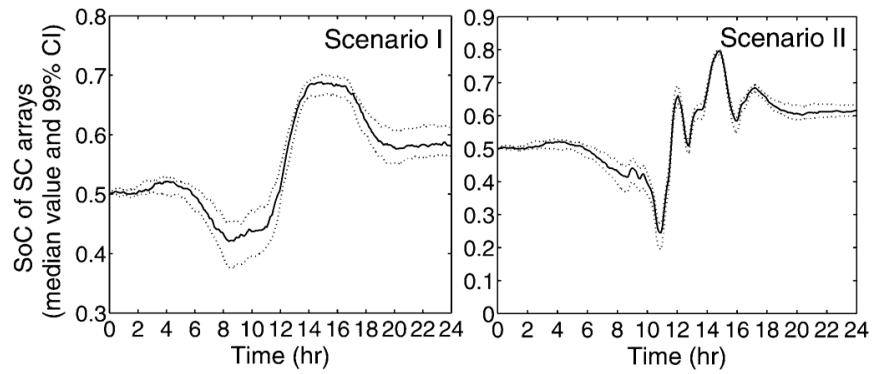

Fig. 8. 24-h $S o C$ of the SC arrays for two different scenarios of forecasting errors in the PV production.
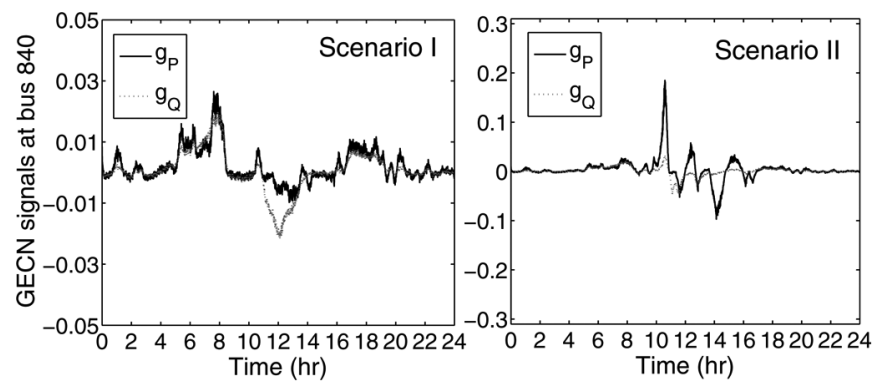

Fig. 9. 24-h GECN signals sent to bus 840 for two different scenarios of forecasting errors in the PV production.

approximation. However, such an assumption is no longer applicable to distribution networks that, when performing voltage control, require to take into account active power injections in addition to reactive power injections.

In the following, we investigate the importance of active versus reactive power-support for voltage control in these specific networks. To this end, we vary the resistance of the lines and we observe the optimal $\Delta P^{*}$ and $\Delta Q^{*}$ that are able to improve the voltage profile. Fig. 10 depicts the optimal active 


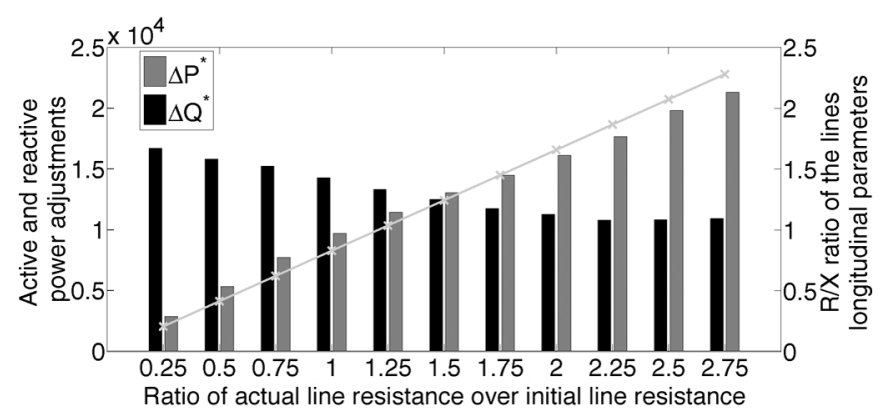

Fig. 10. Optimal active and reactive power adjustments necessary to improve the voltage by $2 \%$ as a function of the line parameters.

and reactive power adjustments for different values of the ratio $R / X$ of the lines. Specifically, the line resistances are varied from 0.25 to 2.75 times their initial value while the line inductances are kept constant. The figure shows the values of the optimal active and reactive power adjustments $\Delta P^{*}$ and $\Delta Q^{*}$ of bus 890 at a specific time-instant. These values are computed in order to improve the network voltage profile by $2 \%$. In the same figure the light-gray line shows the actual $R / X$ of the network lines. One can observe that as the value of the line resistance is increasing, i.e., when the ratio $R / X$ of the lines is increasing, the optimal active power adjustments become more important than the relevant reactive power adjustments.

This observation has two implications. First, as in distribution networks the ratio $R / X$ of the lines is, in general, not negligible, the active power support is necessary when performing primary voltage control. Thus, engaging demand response and ESS control mechanisms in the context of primary voltage control is important. Second, the network characteristics are directly impacting the sizing of the storage devices.

\section{E. Coordination of Heterogeneous Populations for Primary Voltage Control}

This subsection reveals that heterogeneous controllable resources in the network can contribute to primary voltage control, by responding to the same GECN signal. We consider only Scenario II and a large population of elastic thermostatically controlled loads (TCLs) in each network bus (e.g., [15]), in addition to the SC arrays. Specifically, the elastic loads are modeled as refrigerators and represent $20 \%$ of the total peak load in each network bus. We assume that the local controllers of the elastic appliances, as well as the broadcast signals sent to the controllable resources, are as described in [15]. The DNO coordinates with the same signal the loads and the SCs.

In order to quantify the improvement in the network voltage profile due to the coordinated response of the different kinds of resources, we show in Fig. 11 the base case voltage profile and the improved voltage profile obtained when both populations react to the same signal. The maximum improvement in the voltage profile, when all resources are considered, is in the order of $6.5 \%$.

In order to better understand how the different populations contribute to the control action, Fig. 12 shows the active power injected/absorbed by the SC array at bus 840 when the ESSs are the only controllable resources, as well as when TCL and SC
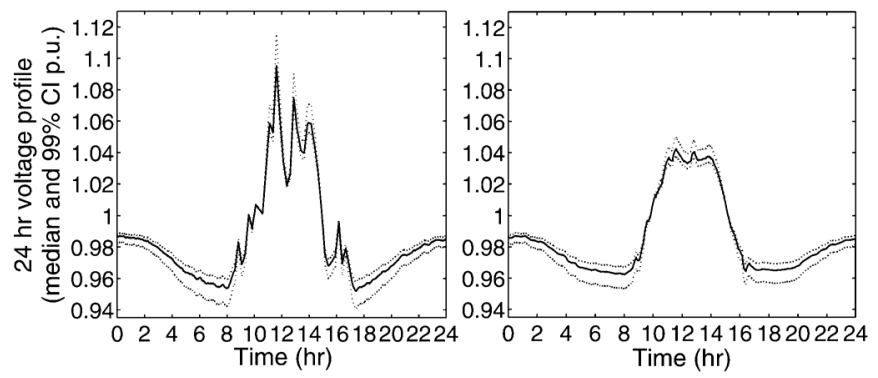

Fig. 11. Base case and improved 24-h network voltage profiles when both SC and TCL respond to GECN.
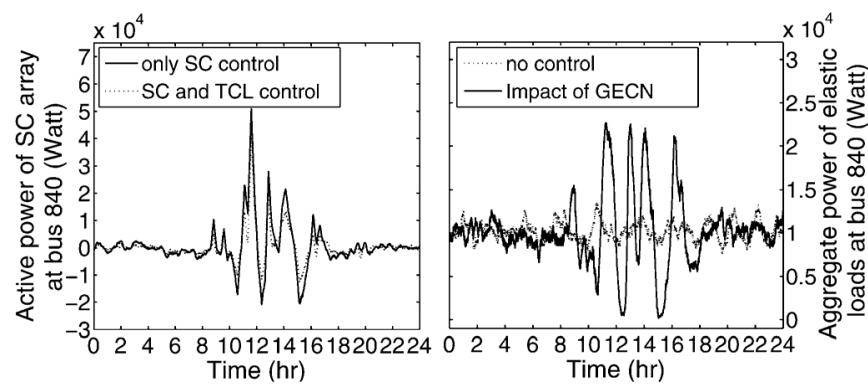

Fig. 12. Active power of the SC array and the elastic appliances when only SC are controlled and when both populations respond to the GECN signals.

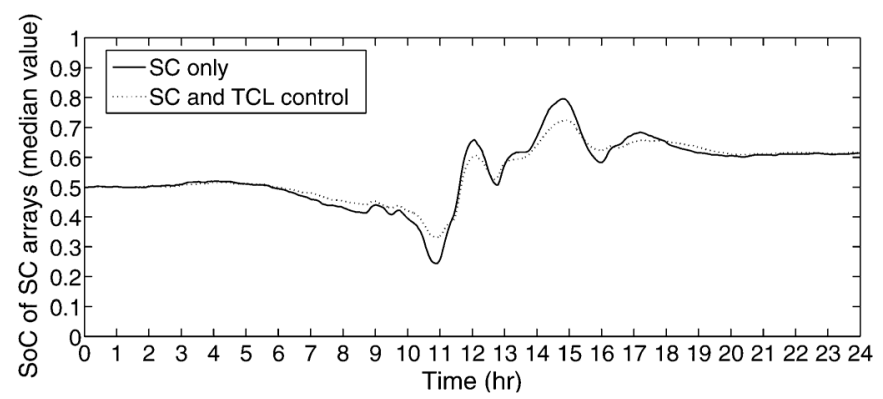

Fig. 13. Median value of the 24-h $S o C$ of the SC arrays when only SC are controlled and when SC and TCL are coordinated.

are coordinated. Also, in this figure the aggregate active power of the elastic loads at bus 840 is depicted for the same cases.

In Fig. 13, the median value $S o C$ of the SC arrays is shown when only SC are controlled (solid line) and when both populations respond to the signals (dashed line). Overall, one can observe that when TCL are included in the control actions the response of the SC is smoothed, i.e., the SC are charged/discharged less when the TCL are also contributing to the voltage control. However, the amount of voltage profile improvement remains almost the same compared to the case of only ESS. This indicates that part of the power that was provided by the ESSs is now substituted by the TCL response. This result is due to the fact that the designed control mechanism requires a given amount of power/energy per bus, which can be provided by any resource connected to the considered bus.

\section{F. Application of GECN to Compensate Fast Voltage Variations: The Case of Load Inrush}

In this section, we show the capability of the proposed control scheme to cope with fast voltage variations originated from a large load inrush. Fig. 14 depicts one hour real measurements of active and reactive power showing the periodic inrush of a large load. During this hour, one can observe that the phenomenon 

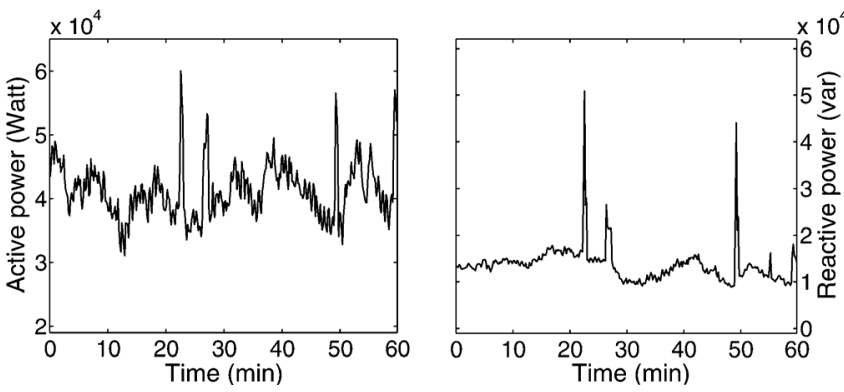

Fig. 14. One-hour measurements of active and reactive power showing the periodic inrush of a large load.

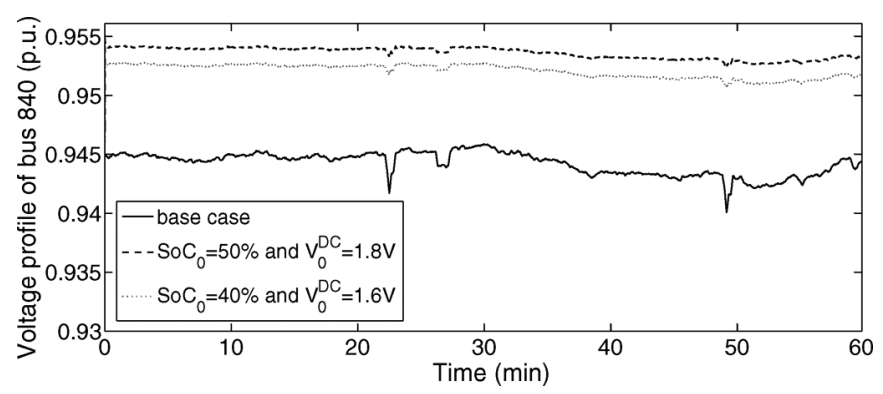

Fig. 15. One-hour voltage profile of bus 840 during a periodic inrush of a large load with and without GECN control.

of load inrush is present both in the active as well as in the reactive power profile. The data have been sampled each second by applying an average filtering of measured quantities.

In order to investigate the performances of the algorithm during voltage sags in the network caused by a load inrush, we apply the proposed voltage control mechanism to control the $\mathrm{SC}$ arrays. In this scenario, the GECN signals are computed by the DNO and sent to the network buses every second. We assume that the load inrush occurs at bus 840 in the most loaded period of the day (i.e., hour 7:15 to 8:15). Also, we consider two different cases. In the first, we assume that the $\mathrm{SC}$ are in their initial state with $S_{o} C=50 \%$ and $V^{\mathrm{DC}}=1.8 \mathrm{~V}$ (SC cell voltage). In the second case we assume that the $\mathrm{SC}$ are already used up to this period of the day and we initialize their state to the state of this specific instant taken from the simulations presented in Section IV-C $\left(S o C=40 \%\right.$ and $\left.V^{\mathrm{DC}}=1.6 \mathrm{~V}\right)$. The results are shown in Fig. 15 where the improvement in the voltage profile is shown for both cases. We can observe that the voltage sags are significantly reduced and the voltage remains within the necessary limits for safe operation. For the sake of completeness we provide in Fig. 16. the signals used to achieve the improvement in the voltage profile in the second case, where the SC arrays are already utilized the moment when the load inrush occurs. In this figure, one can observe how the signals adjust to the specific conditions in the network.

\section{Discussion}

In this work, we propose a control protocol that is able to optimally exploit distributed energy storage, irrespectively of their nature (i.e., TCL or native storage systems) for ADN primary voltage control. In our case, the ESSs are modelled as SC arrays. However, the development of the storage controller, as described in Section III, is not limiting as it is applicable to any

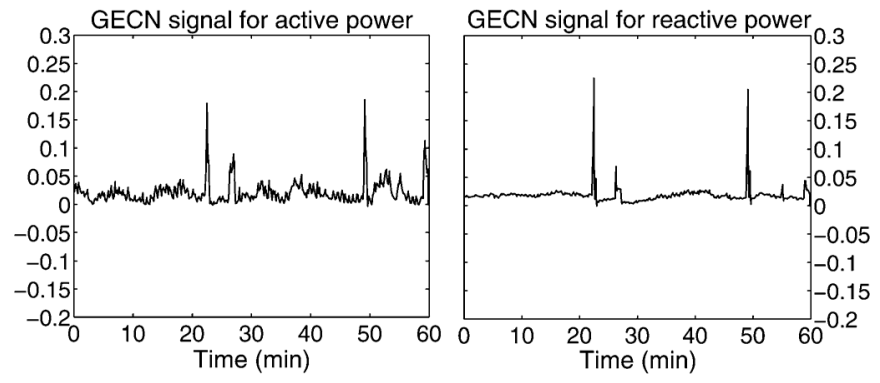

Fig. 16. GECN signals sent to bus 840 during a periodic inrush of a large load.

type of electrochemical-based storage system. Furthermore, we assume that the SC arrays are connected to the MV network buses and are under the propriety of the DNO for the support of the voltage in the network. However, we expect that ESSs, due to their ability to cover a wide spectrum of applications, will be increasingly present in active distribution networks ranging from large units (owned by the DNO or by individual operators) to small, distributed, local storage units (owned by the customers of the grid). In all cases, the proposed algorithm is designed in such a way that if the storage units are able to interpret GECN signals, i.e., are equipped with the storage controller, they can participate in the voltage control actions, as well as in the natural objective of ESSs, namely the local energy balance.

It is also worth observing that the proposed control mechanism has been compared to the use of traditional voltage control actuators composed by OLTCs and shunt capacitors. The simulation results, not provided here for the sake of brevity, have shown 1) the non-effectiveness of shunt capacitors in the period of high PV production and, 2) an increased daily use of OLTCs that causes these components to consume their typical life on a yearly basis (e.g., [36]). Indeed, to the best of our knowledge, voltage regulators were not designed for fast/primary voltage control in ADNs, which inherently requires continuous control actions. For this reason, new literature on the use of distributed storage systems for ADN ancillary services is emerging [18]. However, in case the DNO seeks to use traditional solutions, the GECN mechanism can be used to provide further support to the network, in addition to the DNO's own resources. It is for this reason that the proposed GECN algorithm was initially conceived and designed to coexist with traditional solutions. In fact, in [15], where the GECN algorithm was initially presented, a coordination of elastic appliances and OLTCs is considered, where the OLTC daily operations are limited, to account for their sensitive nature. In the same work, we also mention that reactive power compensators can be directly accounted for in the proposed mechanism. Furthermore, it is important to note that even though the main purpose of GECN is voltage control, as summarized in Section II of the paper, the algorithm also penalizes deviations from the day-ahead scheduled consumption profiles in the network, indirectly performing a sort of power balance that reduces costs of importing energy from the external grid. This is an additional functionality, for example, that cannot be performed by the OLTC or the shunt capacitors.

Another contribution of this work is the description of the inherent flexibility of the proposed control scheme that is capable of achieving similar improvement in the network voltage profile by using solely SC arrays or a combination of ESS and DR 
by sending in all cases a common signal to the resources attached to the network buses. In fact the control mechanism requires a given amount of power/energy per network bus that can be provided by any resource connected to the considered bus. What differs in the various scenarios is the utilization of the different groups of resources. This is supported, for example, by the fact that the SC are charged/discharged less when there is presence of TCL than in the case when there is solely SC control as shown in Fig. 13. Of course, the exact utilization of the different resources depends on several factors such as the availability of different elastic resources in the network, the characteristics of the network itself and the loads/injections profiles. For example, in distribution networks with a high $\mathrm{R} / \mathrm{X}$ ratio, active power management will play a significant role, hence the contribution of elastic loads in the voltage control will be nonnegligible and larger ESSs in terms of energy capacity might be required. Furthermore, due to the design of GECN, which prohibits the operation of the elastic appliances in mini-cycles, it is expected that, in networks where highly volatile uncontrollable generation is present, ESSs will contribute to voltage support more than the elastic loads. Finally, in cases where the availability of the elastic loads or ESSs is limited and the DNO does not want to invest in building new infrastructure, centralized traditional resources might be incorporated in the GECN scheme, such as OLTC or static var compensators (e.g., [15]). In any case, in order to evaluate the availability of the distributed elastic resources in the network and the needs of the network in terms of voltage support, it is advisable that the DNO performs offline studies prior to deploying the proposed scheme. Then, in the case where the needs of the network are not satisfied, it is in the DNO's jurisdiction to decide whether to invest in new dedicated infrastructure, e.g., ESSs, or to coordinate traditional resources with the broadcast signals.

\section{CONCLUSION}

In this paper, we have proposed the extension of a demand-response control mechanism based on low bit-rate broadcast signals, as we previously presented in [15], to control both loads and distributed ESSs. We have verified the inherent flexibility of the proposed control scheme that is capable of controlling nonhomogeneous populations of loads and ESSs to provide specific ancillary services to ADNs.

The proposed control mechanism has been validated by making reference to a typical IEEE 34-node distribution test feeder, appropriately adapted in order to comprise distributed ESSs, a large population of heterogeneous household controllable loads along with nonelastic demand, as well as non-dispatchable power injections. The method is applied in detail to an ADN that uses grid explicit congestion notification (GECN) as a broadcast signal and is also used to size the ESSs. The results show that the proposed storage controller successfully contributes to primary voltage control of distribution networks. Specifically, the capability of controlling the voltage deviations via distributed storage can be up to $6 \%$ of the network's voltage rated value. We have also shown that the proposed mechanism is able to compensate voltage sags associated to the inrush of large loads. In addition, the results indicate that the same GECN control signals are able to sufficiently coordinate different energy resources, as long as the latter are equipped with local controllers that can interpret the signal and respond according to their capabilities. The successful verification of the proposed control scheme makes it a good candidate for dedicated experimental deployment.

\section{APPENDIX}

The use of electrochemical storage systems within the context of ancillary services provided to power distribution networks has been addressed by several contributions to the literature (e.g. [18]). Specifically, in [18] the capability of these systems to provide voltage support to distribution networks is illustrated.

In general, the concerned technologies are represented by battery storage systems. In order to illustrate the potential economical advantages related to the use of supercapacitors versus battery technologies, this section briefly assesses the economical comparison of the two technologies accounting both for the capital and operation investments. The assessment of this last element has been performed using the cost per-cycle and per-kWh.

As far as the capital investment is concerned, we have considered the equivalent annual cost $(E A C)$ defined as the per-year cost of operating a system over its life span [37]:

$$
E A C=\frac{C}{A_{n, i r}}
$$

where $C$ is the invested capital and $A_{n, i r}$ is the known annuity factor. This factor is defined as

$$
A_{n, i r}=\frac{1}{i r}\left(1-\frac{1}{(1+i r)^{n}}\right)
$$

in which $n$ is the lifetime of the energy storage systems in years and $i r$ is the annual interest rate.

Concerning the evaluation of the per-cycle and per-kWh cost, the expected number of cycles, namely the expected lifetime, should be taken into account. The per-cycle $\operatorname{cost}\left(C_{p c}\right)$ is defined as the capital cost $(C)$ of one full cycle of a storage device as [37]:

$$
C_{p c}=\frac{C \cdot D O D \cdot w_{p c}}{N_{c}}
$$

Where $D O D$ is the depth-of-discharge, $N_{c}$ is the number of expected cycles and $w_{p c}$ is the energy delivered by the targeted storage system per cycle.

The comparison is made with reference to SC and Li-ion batteries. The general characteristics of these two technologies are shown in Table IV. To make a fair comparison, $24 \mathrm{SC}$ cells with a rated voltage of $2.7 \mathrm{~V}$ and a total stored energy of $8.59 \mathrm{Wh}$ are used in order to have the same amount of energy as a corresponding Li-ion battery composed of a single $2.7 \mathrm{~V}$ cell with a rated stored energy of $8.6 \mathrm{Wh}$. Also, it is considered that the $D O D$ for both the Li-ion battery and SC equals $100 \%$.

Table IV reports the comparison of both $E A C$ and $C_{p c}$. It can be observed that, by taking advantage of the supercapacitor lifetime, the slightly higher initial capital cost of this technology is largely absorbed by its longer lifetime with a $C_{p c}$ which is lower than the one of the Li-ion batteries. The results reported in Table IV show that supercapacitors are, also from the economic 
TABLE IV

EAC AND PER-CYCLE COST COMPARISON OF SC AND Li-ION BATTERY

\begin{tabular}{|c|c|c|}
\hline & $\mathbf{S C}$ & $\begin{array}{c}\text { Li-ion battery } \\
\text { (M-Type) }\end{array}$ \\
\hline No. of cycles $(D O D=1)$ & $1,000,000$ & 10,000 \\
\hline Capital cost per cell $(\$)$ & 8 (192 for 24 cells $)$ & 100 \\
\hline Energy rated per cell (Wh) & 0.358 & 8.6 \\
\hline Life (years) & 10 & 5 \\
\hline Cost per cycle $(\$ / k W h)$ & 0.022 & 1.16 \\
\hline$E A C(\$)$ & 24.86 & 23.1 \\
\hline
\end{tabular}

point of view, comparable to or even outperforming the standard battery storage systems.

\section{REFERENCES}

[1] J. Lopes, N. Hatziargyriou, J. Mutale, P. Djapic, and N. Jenkins, "Integrating distributed generation into electric power systems: A review of drivers, challenges and opportunities," Electric Power Syst. Res., vol. 77, no. 9, pp. 1189-1203, 2007.

[2] N. Singh, E. Kliokys, H. Feldmann, R. Kussel, R. Chrustowski, and C. Joborowicz, "Power system modelling and analysis in a mixed energy management and distribution management system," IEEE Trans. Power Syst., vol. 13, no. 3, pp. 1143-1149, Aug. 1998.

[3] N.-G. James, Control and Automation of Electrical Power Systems. Hoboken, NJ, USA: CRC, 2006.

[4] T. Senjyu, Y. Miyazato, A. Yona, N. Urasaki, and T. Funabashi, "Optimal distribution voltage control and coordination with distributed generation," IEEE Trans. Power Del., vol. 23, no. 2, pp. 1236-1242, Apr. 2008.

[5] A. Borghetti, M. Bosetti, S. Grillo, S. Massucco, C. Nucci, M. Paolone, and F. Silvestro, "Short-term scheduling and control of active distribution systems with high penetration of renewable resources," IEEE Syst. J., vol. 4, no. 3, pp. 313-322, Sep. 2010.

[6] Q. Zhou and J. Bialek, "Generation curtailment to manage voltage constraints in distribution networks," IET Generat., Transmiss., Distrib., vol. 1, no. 3, pp. 492-498, 2007.

[7] Capacity of Distribution Feeders for Hosting DER TB draft by WG C6.24 CIGRE, 2013

[8] European Network of Transmission System Operators for Electricity (ENTSO-E), Draft Network Code on Demand Connection, Dec. 5, 2012.

[9] M. Arnold and G. Andersson, "Model predictive control of energy storage including uncertain forecasts," in Proc. PSCC, 2011.

[10] S. Koch, F. Barcenas, and G. Andersson, "Using controllable thermal household appliances for wind forecast error reduction," in Proc. IFAC Conf. Control Methodol. Technol. Energy Efficiency, 2010.

[11] H. E. Z. Farag and E. F. El-Saadany, "A novel cooperative protocol for distributed voltage control in active distribution systems," IEEE Trans. Power Syst., vol. 28, no. 2, pp. 1645-1656, May 2013.

[12] H. Farag, E. El-Saadany, and R. Seethapathy, "A two ways communication-based distributed control for voltage regulation in smart distribution feeders," IEEE Trans. Smart Grid, vol. 3, no. 1, pp. 271-281, Mar. 2012

[13] K. Turitsyn, N. Sinitsyn, S. Backhaus, and M. Chertkov, "Robust broadcast-communication control of electric vehicle charging," in Proc. 1st IEEE Int. Conf. Smart Grid Commun. (SmartGridComm), Oct. 2010, pp. 203-207.

[14] S. Bashash and H. Fathy, "Robust demand-side plug-in electric vehicle load control for renewable energy management," in Proc. Amer. Control Conf. (ACC), Jul. 2011, pp. 929-934.

[15] K. Christakou, D.-C. Tomozei, J.-Y. Le Boudec, and M. Paolone, "GECN: Primary voltage control for active distribution networks via real-time demand-response," IEEE Trans. Smart Grid, vol. 5, no. 2, pp. 622-631, Mar. 2014

[16] Q. Zhou and J. Bialek, "Energy storage is a key smart grid element," in Proc. Cigré Symp. Electric Power Syst. Future, Bologna, Italy, Sep. 2011.

[17] M. Nick, M. Hohmann, R. Cherkaoui, and M. Paolone, "On the optimal placement of distributed storage systems for voltage control in active distribution networks," in Proc. Innovative Smart Grid Technologies (ISGT Eur.), Proc. 3rd IEEE PES Int. Conf. Exhib., Oct. 2012, pp. 1-6.
[18] Electric Energy Storage Systems, Cigré Technical Brochure Working Group C6.15, Apr. 2011.

[19] L. Czarnecki and Z. Staroszczyk, "On-line measurement of equivalent parameters for harmonic frequencies of a power distribution system and load," IEEE Trans. Instrum. Meas., vol. 45, no. 2, pp. 467-472, Apr. 1996.

[20] K. Christakou, J. LeBoudec, M. Paolone, and D.-C. Tomozei, "Efficient computation of sensitivity coefficients of node voltages and line currents in unbalanced radial electrical distribution networks," IEEE Trans. Smart Grid, vol. 4, no. 2, pp. 741-750, Jun. 2013.

[21] Q. Zhou and J. Bialek, "Simplified calculation of voltage and loss sensitivity factors in distribution networks," in Proc. 16th Power Syst. Comput. Conf. (PSCC2008), Glasgow, U.K., 2008.

[22] S. Piller, M. Perrin, and A. Jossen, "Methods for state-of-charge determination and their applications," J. Power Sources, vol. 96, no. 1, pp 113-120, 2001.

[23] B. Belvedere, M. Bianchi, A. Borghetti, C. Nucci, M. Paolone, and A. Peretto, "A microcontroller-based power management system for standalone microgrids with hybrid power supply," IEEE Trans. Sustainable Energy, vol. 3, no. 3, pp. 422-431, Jul. 2012.

[24] V. Pop, H. J. Bergveld, P. Notten, and P. P. Regtien, "State-of-the-art of battery state-of-charge determination," Meas. Sci. Technol., vol. 16 , no. 12, p. R93, 2005.

[25] I. Papic, "Simulation model for discharging a lead-acid battery energy storage system for load leveling," IEEE Trans. Energy Convers., vol. 21, no. 2, pp. 608-615, Jun. 2006.

[26] M. Coleman, C. K. Lee, C. Zhu, and W. G. Hurley, "State-of-charge determination from EMF voltage estimation: Using impedance, terminal voltage, current for lead-acid and lithium-ion batteries," IEEE Trans. Ind. Electron., vol. 54, no. 5, pp. 2550-2557, Oct. 2007.

[27] B. Yann Liaw, G. Nagasubramanian, R. G. Jungst, and D. H. Doughty, "Modeling of lithium ion cells a simple equivalent-circuit model approach," Solid State Ionics, vol. 175, no. 1, pp. 835-839, 2004.

[28] S. R. Nelatury and P. Singh, "Equivalent circuit parameters of nickel/metal hydride batteries from sparse impedance measurements," J. Power Sources, vol. 132, no. 1, pp. 309-314, 2004.

[29] A. J. Salkind, P. Singh, A. Cannone, T. Atwater, X. Wang, and D. Reisner, "Impedance modeling of intermediate size lead-acid batteries," J. Power Sources, vol. 116, no. 1, pp. 174-184, 2003.

[30] T. B. Reddy, Linden's Handbook of Batteries. New York, NY, USA: McGraw-Hill, 2011, vol. 4.

[31] R. G. Valle and J. A. P. Lopes, Electric Vehicle Integration Into Modern Power Networks. : Springer, 2012, vol. 2.

[32] L. Zubieta and R. Bonert, "Characterization of double-layer capacitors for power electronics applications," IEEE Trans. Ind. Applicat., vol 36 , no. 1, pp. 199-205, 2000.

[33] D. Torregrossa, M. Bahramipanah, E. Namor, R. Cherkaoui, and M. Paolone, "Improvement of dynamic modeling of supercapacitor by residual charge effects estimation," IEEE Trans. Ind. Electron., vol. 61, no. 3, pp. 1345-1354, 2014.

[34] W. Kersting, "Radial distribution test feeders," in Proc. IEEE Power Eng. Society Winter Meeting, 2001, vol. 2, pp. 908-912.

[35] M. Eremia and M. Shahidehpour, Handbook of Electrical Power System Dynamics: Modeling, Stability, Control. New York, NY, USA: Wiley-IEEE Press, 2013, vol. 92.

[36] C. H. K. W. S. Virayavanich and A. Seiler, "Reliability of on-load tap changers with special consideration of experience with delta connected transformer windings and tropical environmental conditions," in Cigré Paper, 1996, pp. 12-103.

[37] A. Simpson and G. Walker, "Lifecycle costs of ultracapacitors in electric vehicle applications," in Proc. IEEE 33rd Annu. Power Electron. Specialists Conf. (PESC 020, 2002, vol. 2, pp. 1015-1020.

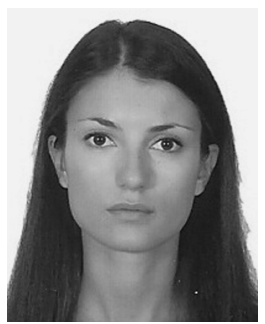

Konstantina Christakou (S'12) was born in Greece, in 1987. She graduated from the National Technical University of Athens, Athens, Greece, in 2010. She is currently pursuing the Ph.D. degree at the École Polytechnique Fédérale de Lausanne (EPFL), Lausanne, Switzerland.

She is currently with EPFL working under the joint supervision of Profs. J.-Y. Le Boudec (LCA2) and M. Paolone (DESL). Her current research interests include control and real-time operation of electrical grids with special reference to power distribution networks. 


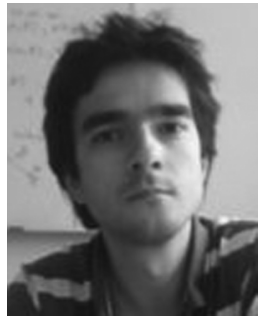

Dan-Cristian Tomozei (M'11) received undergraduate degrees at the École Polytechnique, Paris, France, and received the Ph.D. degree from the University "Pierre et Marie Curie" (UPMC), Paris, France. in 2011

During the Ph.D. degree, he was with the Technicolor Paris Research Lab; he developed distributed algorithms for congestion control and content recommendation in peer-to-peer networks. Since March 2011, he has been a Postdoctoral Researcher at the École Polytechnique Fédérale de Lausanne (EPFL), Switzerland. He is working in the group of Prof. J.-Y. Le Boudec (LCA2) on communication and control mechanisms for the smart grid.

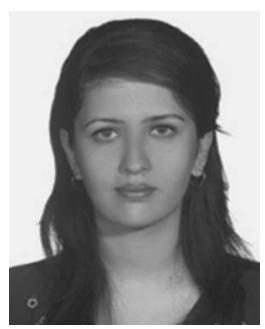

Maryam Bahramipanah was born in Tehran, Iran, in 1984. She received the M.S. degree in electrical engineering from the University of Tehran, Tehran, in 2011. She is currently working toward the Ph.D. degree in power system engineering at the Swiss Federal Institute of Technology (EPFL), Lausanne, Switzerland.

Her research interests refer to the modeling and control of distributed energy-storage systems for the integration of highly volatile renewable energy resources in active distribution networks.

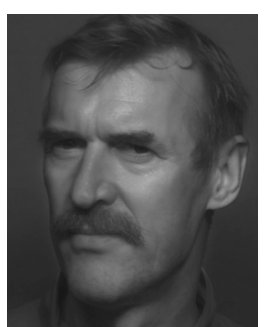

Jean-Yves Le Boudec (F'04) He graduated from École Normale Superieure de Saint-Cloud, Paris, France, and received the Agregation in mathematics in 1980 (rank 4) and the doctorate degree from the University of Rennes, Rennes, France, in 1984

$\mathrm{He}$ is a Full Professor at the Swiss Federal Institute of Technology (EPFL), Lausanne, Switzerland. From 1984 to 1987 he was with INSA/IRISA, Rennes. In 1987, he joined Bell Northern Research, Ottawa, ON, Canada, as a member of scientific staff in the Network and Product Traffic Design Department. In 1988, he joined the IBM Zurich Research Laboratory where he was Manager of the Customer Premises Network Department. In 1994 he joined EPFL as an
Associate Professor. His interests are in the performance and architecture of communication systems. In 1984, he developed analytical models of multiprocessor, multiple bus computers. In 1990, he invented the concept called "MAC emulation" which later became the ATM forum LAN emulation project, and developed the first ATM control point based on OSPF. He also launched public domain software for the interworking of ATM and TCP/IP under Linux. He proposed in 1998 the first solution to the failure propagation that arises from common infrastructures in the Internet. He contributed to network calculus, a recent set of developments that forms a foundation to many traffic control concepts in the internet, and coauthored a book on this topic. He is also the author of the book Performance Evaluation (EPFL Press, 2010).

Prof. Le Boudec received the IEEE Millennium Medal, the Infocom 2005 Best Paper Award, the CommSoc 2008 William R. Bennett Prize and the 2009 ACM Sigmetrics Best Paper award. He is or has been on the program committee or editorial board of many conferences and journals, including Sigcomm, Sigmetrics, Infocom, Performance Evaluation and IEEE/ACM TRANSACTIONS ON NETWORKING.

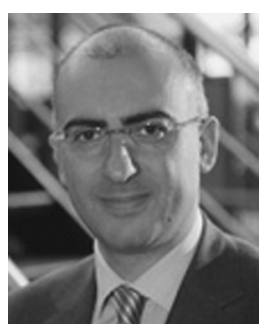

Mario Paolone (SM'10) was born in Italy in 1973. He received the M.Sc. degree (with honors) in electrical engineering and the Ph.D. degree from the University of Bologna, Bologna, Italy, in 1998 and 2002, respectively.

In 2005, he was appointed Researcher in Electric Power Systems at the University of Bologna where he was with the Power Systems Laboratory until 2011. In 2010, he received Associate Professor eligibility from the Politecnico di Milano, Italy. Currently, he is Associate Professor at the Swiss Federal Institute of Technology (EPFL), Lausanne, Switzerland, where he accepted the EOS Holding Chair of Distributed Electrical Systems Laboratory. He is secretary and member of several IEEE and Cigré Working Groups. He was co-chairperson of the Technical Committee of the 9th edition of the International Conference of Power Systems Transients (IPST 2009). His research interests are in power systems with particular reference to real-time monitoring and operation, power system protections, power systems dynamics and power system transients. He is author or coauthor of over 170 scientific papers published in reviewed journals and presented at international conferences.

Prof. Paolone was the recipient of the IEEE EMC Society Technical Achievement Award in 2013 